TecnoLógicas

ISSN-p 0123-7799

ISSN-e 2256-5337

Vol. 23, No. 48, pp. 119-141

Mayo-agosto de 2020

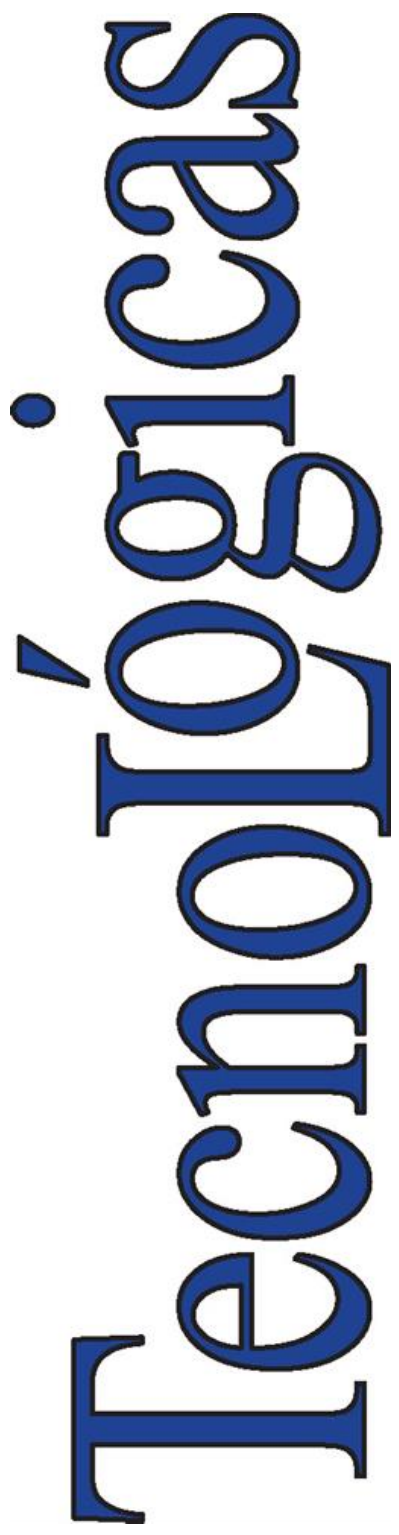

(C) Instituto Tecnológico Metropolitano Este trabajo está licenciado bajo una Licencia Internacional Creative Commons Atribución (CC BY-NC-SA)
Artículo de Investigación/Research Article

\section{Estudio técnico-económico de dos tecnologías de producción de biodiesel a partir de aceite de soya empleando el simulador superpro designer}

\section{Techno-Economic Study of Two Biodiesel Production Technologies from Soybean 0il Using Superpro Designer Simulator}

\author{
Leonardo Campos-Ramírez ${ }^{(D)}$ \\ Amaury Pérez-Sánchez (D) $\mathbf{O}^{2}$, \\ Aylín Benítez-Legráid ${ }^{3} \mathrm{y}$ \\ Isnel Benítez ${ }^{(D)} 4$ \\ Recibido: 11 de diciembre de 2019 \\ Aceptado: 31 de marzo de 2020
}

\section{Cómo citar / How to cite}

L. Campos-Ramírez, A. Pérez-Sánchez, A. Benítez-Legrá, I. Benítez, "Estudio técnico-económico de dos tecnologías de producción de biodiesel a partir de aceite de soya empleando el simulador superpro designer", TecnoLógicas, vol. 23, no. 48, pp. 119-141, 2020.

https://doi.org/10.22430/22565337.1568

1 PhD. en Ciencias Técnicas, Centro de Ciencias y Tecnologías Agropecuarias (CCTA), Universidad Estatal del Norte Fluminense, Río de Janeiro-Brasil, leocampito1969@gmail.com

2 Ingeniero Químico, Departamento de Ingeniería Química, Facultad de Ciencias Aplicadas, Universidad de Camagüey, Camagüey-Cuba, amauryps@nauta.cu

3 Ingeniero Químico, Departamento de Proyectos, Instituto de Proyectos Azucareros, Camagüey- Cuba, aylin.benitez@iproyazcm.azcuba.cu

4 PhD. en Ciencias Técnicas, Departamento de Ingeniería Química, Facultad de Ciencias Aplicadas, Universidad de Camagüey, Camagüey-Cuba, isnel.benites@reduc.edu.cu 


\section{Resumen}

En el presente trabajo se efectuó un estudio técnico-económico preliminar de dos propuestas tecnológicas de producción de biodiesel empleando aceite de soya como materia prima principal y bajo las condiciones económicas actuales de Brasil. Para eso se utiliza el simulador SuperPro Designer ${ }^{\circledR}$ v.8.5. En la primera tecnología propuesta (Caso Base) se obtiene biodiesel refinado y glicerol crudo, mientras que en la segunda (Variante) también se produce biodiesel refinado, y el glicerol crudo es purificado hasta una pureza final de $99,75 \%$. Se efectuó un estudio de sensibilidad consistente en 12 corridas mediante el cual se evaluó la influencia de cinco variables de entrada sobre tres indicadores económicos: Valor Actual Neto (VAN), Tasa Interna de Retorno (TIR) y Periodo de Recuperación de la Inversión (PRI). Se deben invertir USD $\$ 18,5$ millones y USD $\$ 21,4$ millones para construir el Caso Base y la Variante, respectivamente. Los resultados del VAN, TIR y PRI para el Caso Base fueron de USD $\$ 17444000,33,83 \%$ y 2,54 años respectivamente, mientras que los valores obtenidos de estos 3 indicadores para la Variante fueron de USD $\$ 22577000,38,05 \%$ y 2,26 años respectivamente, cosa que indica que la Variante constituye la propuesta tecnológica más rentable. La introducción de operaciones de purificación de glicerol incrementa los indicadores económicos y de rentabilidad de la planta de producción de biodiesel. El estudio de sensibilidad permitió obtener ecuaciones que establecen la correlación estadística existente entre cinco variables de entrada y tres de salida. Se empleó el software Statgraphics Centurion ${ }^{\circledR}$ versión XVI para el procesamiento estadístico de los resultados obtenidos.

\section{Palabras clave}

Aceite de soya, análisis de sensibilidad, biodiesel, glicerol, SuperPro Designer.

\section{Abstract}

In this work, a preliminary technical-economic study of two technological proposals of biodiesel production was carried out employing soybean oil as the main raw material and under the current economic conditions of Brazil. For that the simulator SuperPro Designer ${ }^{\circledR}$ v.8.5 is used. In the first technology (Base Case) refined biodiesel and crude glycerol are obtained, while in the second (Variant) refined biodiesel is also produced, and the crude glycerol is purified to a final purity of $99.75 \%$. A sensitivity study consisting of 12 runs was carried out, through which the influence of five input variables on three economic indicators was evaluated: Net Present Value (NPV), Internal Rate of Return (IRR) and Payback Time (PT). USD \$ 18.5 million and USD \$ 21.4 million must be invested to erect the Base Case and the Variant, respectively. The results of the NPV, IRR and PT for the Base Case were USD \$ $17,444,000,33.83 \%$ and 2.54 years respectively, while the values obtained from these 3 indicators for the Variant were USD $\$ 22,577,000$, 38.05\% and 2.26 years respectively, which indicate that the Variant is the most cost-effective technology. The introduction of glycerol purification operations increases the economic and profitability indicators of the biodiesel production plant. The sensitivity study allowed obtaining equations that establish the statistical correlation between five input variables and three output parameters. Statgraphics Centurion ${ }^{\circledR}$ software version XVI was used for the statistical processing of the results obtained.

\section{Keywords}

Soybean oil, sensitivity analysis, biodiesel, glycerol, SuperPro Designer. 


\section{INTRODUCCIÓN}

Los aceites vegetales como combustibles fueron utilizados por primera vez en 1900, siendo Rudolph Diesel quien los utilizara en su motor de ignición-compresión y quien predijera el uso futuro de biocombustibles [1].

El biodiesel es un combustible líquido obtenido por procesos químicos a partir de aceites vegetales o grasas animales y un alcohol, el cual puede ser empleado en motores diésel, ya sea solo o mezclado con combustible diésel.

El biodiesel se produce mediante una reacción de transesterificación, en la cual se convierte un éster (aceite vegetal o grasa animal) en una mezcla de ésteres de ácidos grasos que conforman el aceite (o grasa). El biodiesel se obtiene a partir de la purificación de la mezcla de ésteres metílicos de ácidos grasos (EMAG).

Usualmente se emplea un catalizador para acelerar la reacción. De acuerdo con el catalizador usado, la transesterificación puede ser básica, ácida o enzimática, siendo la primera la más empleada.

El contenido de ácidos grasos libres, agua y sustancias no saponificables son parámetros importantes para lograr una elevada eficiencia de conversión en la reacción de transesterificación [2].

En la actualidad, se obtiene glicerina (o glicerol) como sub-producto de la producción de biodiesel, el cual es purificado para eliminar los contaminantes presentes, fundamentalmente el catalizador y el alcohol, y ser posteriormente comercializado.

La separación de los productos de reacción tiene lugar mediante decantación: la mezcla de EMAG se separa de la glicerina, formando dos fases, ya que presentan densidades diferentes; las dos fases empiezan a formarse inmediatamente después de que se detiene la agitación de la mezcla. Debido a la diferencia de su afinidad química, la mayoría de catalizador y el alcohol en exceso se concentrarán en la fase pesada o de fondo (glicerina), mientras que la mayoría de los mono-, di- y triglicéridos (EMAG) se concentrarán en la fase ligera o superior.

La simulación, desde el punto de vista de la ingeniería química, es la solución de las ecuaciones de balance de materia y energía para procesos químicos en estado estacionario o dinámico, así como del dimensionamiento y la obtención de costos de los equipos involucrados en un proceso determinado [3]. La simulación y modelación de proceso no es más que la aplicación de herramientas de software para analizar operaciones unitarias individuales (o etapas de proceso) y su relación con el proceso global. La simulación de proceso se define entonces como la utilización de recursos de software para desarrollar un modelo matemático para la construcción de un modelo representativo de un proceso químico, con el fin de comprender el comportamiento real durante la operación de la planta [4].

Los simuladores constituyen una herramienta poderosa para los ingenieros, con el fin de diseñar, evaluar u optimizar un determinado proceso. Entre los simuladores más empleados en la actualidad se encuentra el SuperPro Designer $^{\circledR}$, el cual se ha empleado para simular diferentes aplicaciones y procesos químicos, estos son: la digestión anaerobia de materia orgánica compleja [5], la desmineralización parcial de la leche de vaca [6], la producción de oleorresina a partir de Capsicum frutescens [7], la extracción de antocianina y producción de etanol a partir de maíz en un proceso de molienda en seco modificado [8], la producción de etanol a partir de Ipomoea batatas [9] y la producción de biodiesel a partir de aceite de soya y metanol [10].

Son varios los autores que han simulado procesos de producción de biodiesel a diversas escalas para determinar su viabilidad técnica, económica y medioambiental. 
Estudio técnico-económico de dos tecnologías de producción de biodiesel a partir de aceite de soya empleando el simulador superpro designer

En este sentido, [11] realizó la simulación de una planta de producción y purificación de biodiesel a partir de aceite de palma y etanol usando el simulador HYSYS 3.2, presentando como resultado el diagrama de flujo del proceso junto con las condiciones detalladas de operación, así como el dimensionamiento de los equipos involucrados en el proceso. [12] utilizaron el simulador HYSYS para simular tres procesos continuos de producción de biodiesel con una capacidad de 40000 t/año, incluyendo el proceso convencional catalizado por álcalis usando aceite vegetal fresco y residual, y un proceso con metanol supercrítico utilizando aceite vegetal residual como materia prima. Asimismo, [13] desarrolló la simulación de una planta industrial de producción de biodiesel en condiciones supercríticas empleando una relación molar metanol/triglicéridos de 9:1 y una temperatura de reacción de $400{ }^{\circ} \mathrm{C}$, empleando para ello el simulador Aspen Plus® 2006. [14] llevó a cabo la simulación del proceso de producción de biodiesel bajo condiciones súper-críticas mediante el simulador Aspen Plus ${ }^{\circledR}$. Para ello basaron las condiciones de operación en el sistema experimental utilizado a escala piloto. [15] efectuaron la simulación del proceso de producción de biodiesel a escala industrial en el simulador $\mathrm{PRO} / \mathrm{II}^{\circledR}$ mediante la catálisis básica homogénea, empleando el aceite de soya crudo y el aceite de palma crudo como materias primas, además del etanol. Por otro lado, [16] desarrollaron un diseño conceptual y simulación del proceso de producción de biodiesel a partir del aceite de Jatropha curcas, mediante la simulación de sus etapas en el software HYSYS $^{\circledR}$. Para este estudio, se trabajó con $11000 \mathrm{~kg} / \mathrm{h}$ de aceite como materia prima, se tuvo en cuenta una etapa de esterificación debido a los altos contenidos de ácidos grasos libres (AGL) presentes en este aceite $y$ se emplearon diversos modelos termodinámicos, lo cual permitió obtener un biocombustible con una composición mayor al $98 \%$ (masa) en metilésteres. Además, en [17] diseñaron y simularon un proceso de transesterificación continúo catalizado por álcalis con una capacidad de producción de biodiesel de 8000 t/año, utilizando aceite de semilla de Jatropha curcas como materia prima, mediante el empleo del simulador HYSYS $^{\circledR}$. En otro trabajo, [18] emplearon el simulador SuperPro Designer ${ }^{\circledR}$ v. 7.0 para simular el proceso de producción de biodiesel a partir de aceite de Jatropha curcas. Por último, [19] emplearon el simulador Aspen HYSYS $^{\circledR}$ para simular el proceso de producción de biodiesel a partir de aceite de cocina residual, empleando un reactor de membrana.

En la actualidad los aceites vegetales representan el $90 \%$ de la materia prima empleada para producir biodiesel a escala mundial [20]. El aceite de soya puede ser empleado en la industria alimenticia o como materia prima para producir lubricantes, así como también en la producción de biodiesel. Actualmente, la soya es una de las materias primas más empleadas en la producción de biodiesel a escala mundial [21]-[23], en donde la conversión del aceite de soya en éster se lleva a cabo típicamente mediante el proceso de transesterificación, la cual es la tecnología líder utilizada para producir biodiesel [20].

En los últimos años la producción de biodiesel empleando diversas materias primas y procesos ha sido estudiada extensivamente [24]-[30].

La producción anual de biodiesel en Brasil alcanzó los 5,4 billones de litros en el año 2018, ubicándose segunda solo por detrás de la estadounidense [31]. El aceite de soya es la principal materia prima en el sector de producción de biodiesel brasileño, con un $70 \%$ del total [32], cifra que se justifica por su importancia en el agronegocio y el desarrollo apropiado de aspectos agronómicos, industriales y logísticos [20].

Alentado por políticas públicas $\mathrm{y}$ acuerdos internacionales basados en la 

simulador superpro designer

promoción del uso de combustibles renovables y la reducción de las emisiones de gases de efecto invernadero, la demanda de biodiesel está vaticinada a aumentar para poder cumplir con las regulaciones globales. En este sentido, Brasil puede jugar un papel importante como suministrador neto de biodiesel a escala mundial debido a su disponibilidad de tierra y elevadas reservas de materias primas [20].

El objetivo del presente trabajo consiste en determinar los principales indicadores técnico-económicos de dos propuestas tecnológicas de producción de biodiesel a partir de aceite de soya, empleando para ello el simulador SuperPro Designer ${ }^{\circledR}$ v. 8.5, con el fin de conocer si ambas son rentables y factibles desde el punto de vista económico bajo las condiciones económicas actuales de Brasil. También se efectúa un estudio de sensibilidad para determinar la correlación estadística existente entre cinco variables iniciales o de entrada y tres parámetros de salida; por último, se realiza una comparación entre los diversos indicadores económicos obtenidos para ambas tecnologías, con el fin de establecer la más rentable.

\section{MATERIALES Y MÉTODOS}

\subsection{Descripción del proceso de producción de biodiesel sin incluir operaciones de purificación del glicerol (Caso Base)}

El proceso de producción de biodiesel (sin incluir etapas de purificación de glicerol) se inicia (Fig. 2) con la formación del catalizador etóxido de sodio $\left(\mathrm{CH}_{3} \mathrm{CH}_{2} \mathrm{NaO}\right)$, haciendo reaccionar $0,36 \mathrm{t}$ de hidróxido de sodio $(\mathrm{NaOH})$ con 1,5 t de etanol $\left(\mathrm{CH}_{3} \mathrm{CH}_{2} \mathrm{OH}\right)$ mediante (1).
La reacción ocurre a $60{ }^{\circ} \mathrm{C}$ de temperatura y por espacio de 30 minutos en un tanque enchaquetado provisto de agitación de $3 \mathrm{~m}^{3}$ de capacidad $\mathrm{y}$ construido de acero inoxidable 316 (Tanque 1), mientras que se emplea una cantidad inicial de $\mathrm{NaOH}$ igual al $1 \%$ de la cantidad de aceite de soya a consumir [15].

De forma simultánea al proceso de formación del etóxido, en otra área de la planta se vierten 11,52 t de etanol y $36,15 \mathrm{t}$ de aceite de soya en sendos tanques cilíndricos verticales de acero al carbono de 18 y $40 \mathrm{~m}^{3}$ de capacidad respectivamente (Tanques 1 y 2), y se mantienen en estos recipientes por espacio de 30 minutos.

Cumplido este tiempo se bombea todo el aceite de soya y etanol contenido en los tanques hacia dos recipientes cilíndricos verticales de acero al carbono de $40 \mathrm{~m}^{3}$ de capacidad, los cuales están enchaquetados y provistos de agitación (R1 y R2), para, acto seguido, bombear hacia estos mismos reactores la mitad (50\%) del volumen de etóxido de sodio previamente obtenido en el Tanque 1. Una vez puestos en contacto el aceite de soya, el etanol y el etóxido, comienza el proceso de formación de biodiesel (reacción de transesterificación), que debe ser controlado a $60{ }^{\circ} \mathrm{C}$ por 60 minutos aproximadamente, para lograr una conversión superior al $95 \%$.

A continuación, se muestra la reacción de transesterificación (Fig. 1). Una vez concluida la reacción de transesterificación en los reactores 1 y 2 , se procede a bombear el contenido de los mismos hacia un primer decantador (Decantador 1) de $60 \mathrm{~m}^{3}$ de capacidad, en donde ocurre una separación de fases; obteniéndose por el tope, una fase ligera rica en biodiesel, mientras que por el fondo se obtiene una fase pesada rica en glicerol y alcohol etílico.

$$
\mathrm{CH}_{3} \mathrm{CH}_{2} \mathrm{OH}+\mathrm{NaOH} \rightarrow \mathrm{CH}_{3} \mathrm{CH}_{2} \mathrm{NaO}+\mathrm{H}_{2} \mathrm{O}
$$


Estudio técnico-económico de dos tecnologías de producción de biodiesel a partir de aceite de soya empleando el simulador superpro designer

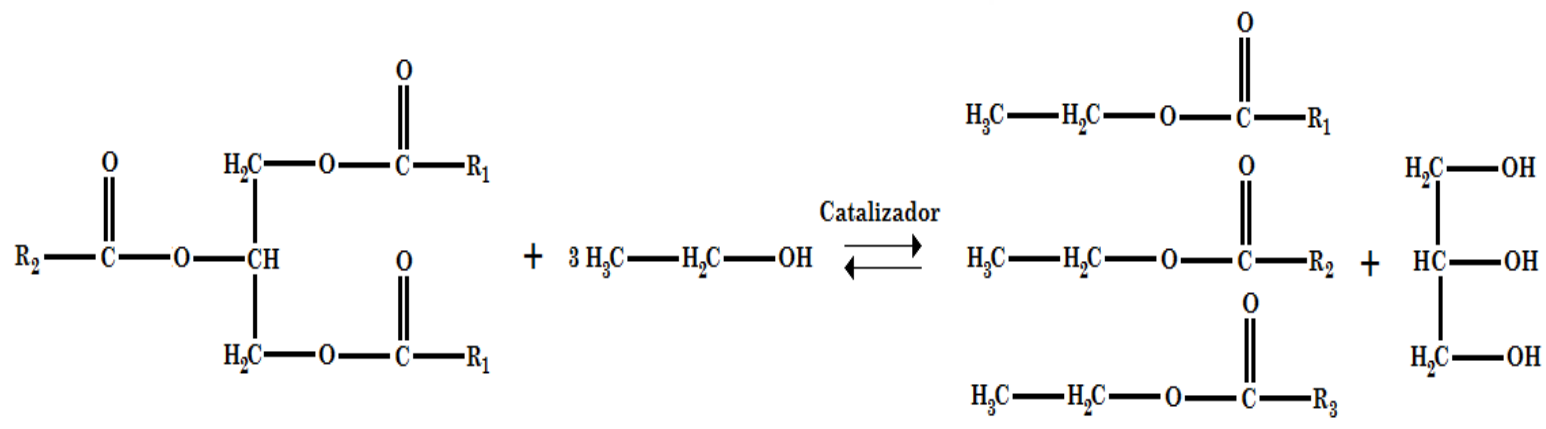

Fig. 1. Reacción de transesterificación empleando etanol

Fuente: elaboración propia.

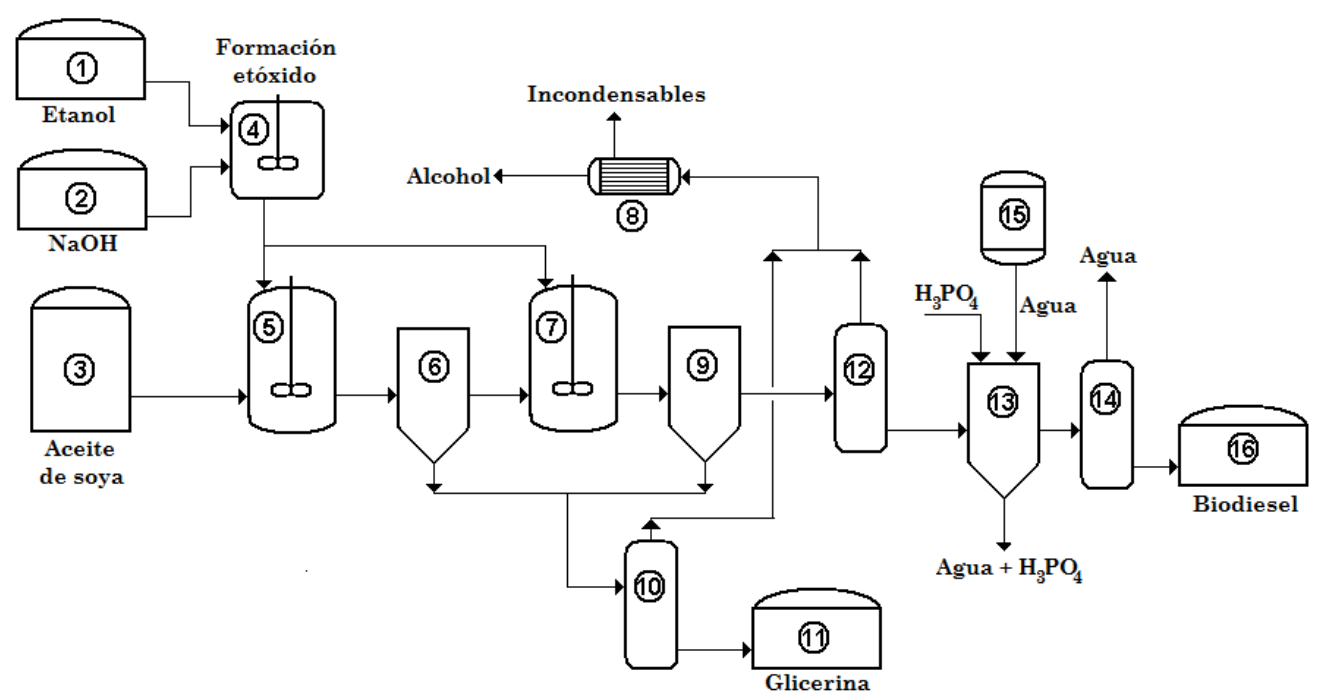

\begin{tabular}{|ll|}
\hline \multicolumn{1}{|c|}{ LEYENDA } \\
1 - Tanque recepción etanol & 9 - Decantador 2 \\
2 - Tanque recepción NaOH & 10 - Evaporador 1 \\
3 - Tanque recepción aceite de soya & 11 - Tanque almacenamiento glicerina cruda \\
4 - Reactor de formación etóxido & 12 - Evaporador 2 \\
5 - Reactor de transesterificación 1 & 13 - Tanque de lavado biodiesel \\
6 - Decantador & 14 - Evaporador 3 \\
7 - Reactor de transesterificación 2 & 15 - Tanque recepción agua de retorno \\
8- Condensador & 16 - Tanque almacenamiento biodiesel \\
\hline
\end{tabular}

Fig. 2. Diagrama de flujo del Caso Base

Fuente: elaboración propia.

El proceso de decantación (separación de fases) en este equipo dura aproximadamente 2 horas. La fase ligera es enviada hacia dos reactores secundarios de $40 \mathrm{~m}^{3}$ de capacidad cada uno (reactores de transesterificación 3 y 4), también enchaquetados y provistos de agitación, a los cuales se le agrega además el volumen de etóxido de sodio que quedó remanente en el primer tanque (50\%). Dentro de estos reactores se convierte en biodiesel el aceite de soya que quedó sin reaccionar en los primeros dos reactores, ocurriendo esta reacción también a $60{ }^{\circ} \mathrm{C}$ de temperatura por 60 minutos aproximadamente, obteniéndose por tanto una conversión global de aceite en biodiesel cercana al $99 \%$. Una vez concluida la reacción de 

simulador superpro designer

transesterificación en los reactores 3 y 4 , la mezcla resultante es bombeada hacia un segundo decantador de $60 \mathrm{~m}^{3}$ de capacidad (Decantador 2), en donde ocurre una segunda decantación por espacio de 2 horas. De esta última, se obtiene por el tope de este equipo una fase ligera rica en biodiesel, mientras que por el fondo se obtiene una fase pesada rica en etanol y glicerol. La fase ligera es enviada hacia un primer evaporador de película descendente (Evaporador 1), en el cual se lleva a cabo la concentración del biodiesel obtenido, mientras que la fase pesada es mezclada con la fase pesada obtenida en el primer decantador, y la corriente resultante obtenida, la cual es rica en etanol y glicerol, es enviada hacia un segundo evaporador de película descendente (Evaporador 2), con el fin de concentrar el glicerol contenido en esta. Los vapores obtenidos tanto en el Evaporador 1 como en el Evaporador 2, que contienen una elevada concentración de etanol, son mezclados entre sí, y la corriente resultante es dirigida a continuación hacia un intercambiador de calor de tubo $y$ coraza (Condensador), para llevar a cabo la condensación del etanol contenido en la misma, obteniéndose a la salida de este equipo una corriente líquida con un $97 \%$ de alcohol etílico. La corriente líquida obtenida a la salida del Evaporador 2, la cual contiene glicerol con un $74 \%$ de pureza, es enviada hacia un tanque cilíndrico vertical de acero al carbono de 8 $\mathrm{m}^{3}$ de capacidad, para su posterior venta a granel.

Por su parte, la corriente líquida obtenida en el Evaporador 1, la cual contiene biodiesel con un $90 \%$ de pureza, es enviada hacia un tanque de $25 \mathrm{~m}^{3}$ de capacidad en donde se deja enfriar hasta temperatura ambiente, para ser luego bombeada hacia un tanque decantador (Decantador 3) de $100 \mathrm{~m}^{3}$ de volumen, en el cual también se agrega ácido fosfórico al $85 \%$ de pureza y agua, esta última con el fin de lavar y purificar el biodiesel.
Este lavado transcurre en un lapso de 2 horas, obteniéndose al final una fase ligera rica en biodiesel en el tope, y una fase pesada conteniendo mayormente agua en el fondo.

La corriente del tope es enviada a continuación hacia un tercer y último evaporador de película descendente (Evaporador 3), en donde ocurre la concentración y purificación final del biodiesel, obteniéndose a la salida una corriente líquida conteniendo biodiesel con un $96,5 \%$ de pureza, la cual posteriormente es enviada hacia dos tanques de $20 \mathrm{~m}^{3}$ de capacidad para su posterior comercialización. La fase pesada (o fondo) obtenida en el Decantador 3 es enviada hacia la planta de tratamiento de residuales de la planta, mientras que la corriente de vapor de agua obtenida en el Evaporador 3 es vertida hacia la atmósfera. Se pudiera evaluar la reutilización de este vapor de agua residual como agente calefactor en alguna de las etapas que requieren de este servicio, tales como la formación de etóxido y la transesterificación, ya que se obtiene saturado a una temperatura de $118{ }^{\circ} \mathrm{C}$ (entalpía: 2703,01 kJ/kg [33]), cosa que mejoraría la integración $\mathrm{y}$ eficiencia energética del proceso de producción global. El condensado obtenido al emplear este vapor de agua en el área que lo requiera bien puede ser reutilizado entonces como agua de alimentación en la generación de vapor.

\subsection{Descripción del proceso de producción de biodiesel considerando operaciones de purificación del glicerol (Variante)}

El glicerol crudo obtenido a la salida del Evaporador 2, el cual presenta una pureza cercana al $70 \%$, se envía a un tanque de recepción de $8 \mathrm{~m}^{3}$ de capacidad, en donde es almacenado por 30 minutos aproximadamente. El contenido total de este recipiente es bombeado a continuación hacia un tanque enchaquetado provisto de 

simulador superpro designer

agitación de $10 \mathrm{~m}^{3}$ de capacidad, en donde se le agrega una solución de ácido fosfórico al $85 \%$, además de agua, con el fin de neutralizar el hidróxido de sodio y otras sales y compuestos presentes en esta corriente, además de lavar el glicerol. La operación de neutralización demora aproximadamente 1 hora, formándose el compuesto fosfato de sodio $\left(\mathrm{Na}_{3} \mathrm{PO}_{4}\right)$ a partir de la reacción entre el hidróxido de sodio $(\mathrm{NaOH})$ y el ácido fosfórico $\left(\mathrm{H}_{3} \mathrm{PO}_{4}\right)$.

La mezcla resultante de la operación de neutralización, cuyo contenido consta fundamentalmente de glicerol, ácido fosfórico, agua y etanol, es enviada hacia una centrifuga decantadora de $150 \mathrm{~m}^{3 / h}$ de capacidad. De este equipo se obtiene una corriente aceitosa conteniendo glicerol con un $80 \%$ de pureza, y una corriente acuosa conteniendo fundamentalmente agua, etanol y etóxido de sodio. Esta última corriente es enviada hacia la planta de tratamiento de residuales de la fábrica, mientras que la corriente aceitosa es alimentada hacia una columna de destilación al vacío compuesta por 69 etapas y operando a una relación de reflujo $R / R_{\text {min }}$ de 1,50 . En este equipo se obtiene por el tope una corriente compuesta fundamentalmente por ácido fosfórico y agua, la cual es vertida hacia la planta de tratamiento de residuales, mientras que por el fondo de la columna se obtiene una corriente rica en glicerol (91,2 \% de pureza) la cual es enviada hacia tres columnas de carbón activado para proceder a su purificación final. A la salida de las columnas de carbón activado se obtiene una corriente conteniendo glicerol al $99,75 \%$ (glicerol refinado), la cual es almacenada finalmente en un tanque de recepción de $8 \mathrm{~m}^{3}$ de capacidad, para $\mathrm{su}$ posterior comercialización (Fig. 3).

Para el establecimiento del proceso de producción de biodiesel a partir de aceite de soya, así como también para la selección de algunas de sus variables de operación y equipos, tanto para el Caso Base como la Variante, se procedió a consultar diferentes bibliografías [10], [34]-[39].

\subsection{Composición química del aceite de soya}

El aceite de soya empleado en la simulación presenta la siguiente composición química [40] (Tabla 1):

Tabla 1. Composición química del aceite de soya empleado en la simulación

\begin{tabular}{cc} 
& Fuente: [40] \\
\hline Compuesto & Composición (\%) \\
\hline Ácido palmítico & 9,7 \\
Ácido esteárico & 3,0 \\
Ácido mirístico & 0,1 \\
Ácido oleico & 25,4 \\
Ácido palmitoleico & 0,2 \\
Ácido linoleico & 55,0 \\
Acido alfa-linolénico & 6,0 \\
Ácido araquídico & 0,6 \\
\hline
\end{tabular}


Estudio técnico-económico de dos tecnologías de producción de biodiesel a partir de aceite de soya empleando el simulador superpro designer

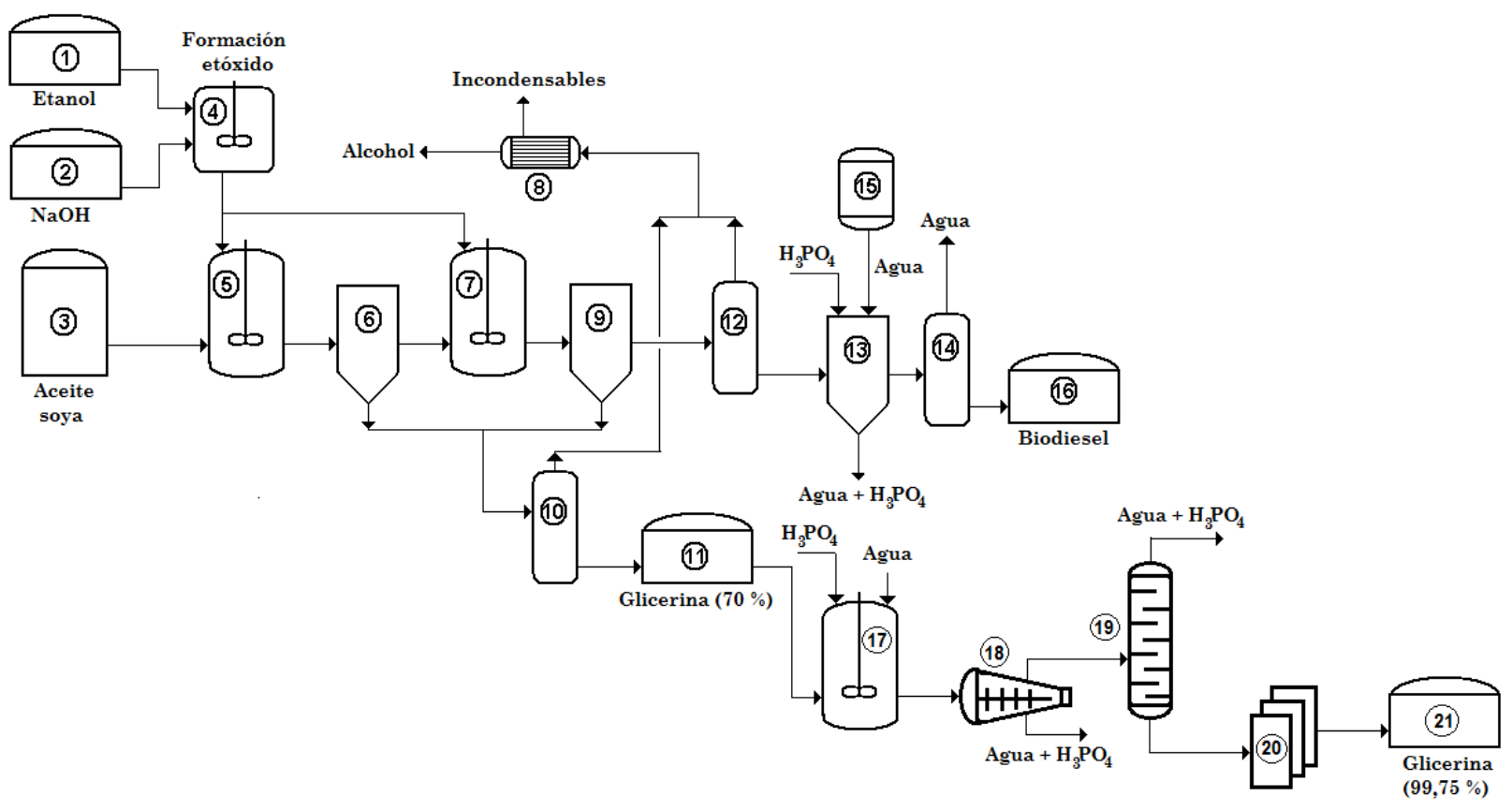

\begin{tabular}{|lll|}
\hline & \multicolumn{1}{c|}{ LEYENDA } \\
1 - Tanque recepción etanol & 9 - Decantador 2 & \\
2 - Tanque recepción NaOH & 10 - Evaporador 1 & - Tanque lavador \\
3 - Tanque recepción aceite de soya & 11 - Tanque almacenamiento glicerina cruda & 19 - Columna de destilación al vacío \\
4 - Reactor de formación etóxido & 12 - Evaporador 2 & 20 - Columna de carbón activado \\
5 - Reactor de transesterificación 1 & 13 - Tanque de lavado biodiesel & 21 - Tanque almacenamiento glicerol purificado \\
6 - Decantador 1 & 14 - Evaporador 3 & \\
7 - Reactor de transesterificación 2 & 15 - Tanque recepción agua de retorno & \\
8 - Condensador & 16 - Tanque almacenamiento biodiesel & \\
\hline
\end{tabular}

Fig. 3. Diagrama de flujo de la Variante. Fuente: elaboración propia.

\subsection{Simulación del Caso Base en el simulador SuperPro Designer ${ }^{\circledast}$}

Se empleó el simulador profesional de procesos SuperPro Designer ${ }^{\circledR}$ v.8.5 para simular el proceso de producción del biodiesel sin incluir operaciones de purificación de glicerol (Caso Base), con el fin de aplicar las herramientas de balance de masa y energía, diseño de equipamiento y cálculos económicos contenidos en el mismo al proceso bajo estudio.

La simulación del Caso Base en el simulador SuperPro Designer ${ }^{\circledR}$ permitió obtener valores y resultados de un gran número de parámetros técnico-económicos de importancia para este proceso de producción, entre los cuales se puede mencionar VAN, TIR, PRI, costos fijos, capital de trabajo, costo unitario de producción, margen bruto y neto, \% de retorno de la inversión, entre otros (Fig. 4).

Para efectuar la simulación del Caso Base se consideró un tiempo de construcción de la planta de 18 meses, con tres meses para realizar la arrancada $y$ puesta en marcha. Se tomó en cuenta un tiempo de vida del proyecto de 15 años, considerando que la planta producirá a un $85 \%$ de capacidad durante los cinco primeros años, para luego producir al $88 \%$ por dos años, al $90 \%$ por cuatro años más, y al $95 \%$ por los restantes cuatro años. El VAN fue determinado para una tasa de interés del $11 \%$, y se consideró un $32 \%$ de impuestos por concepto de ingresos [32]. 
Estudio técnico-económico de dos tecnologías de producción de biodiesel a partir de aceite de soya empleando el simulador superpro designer

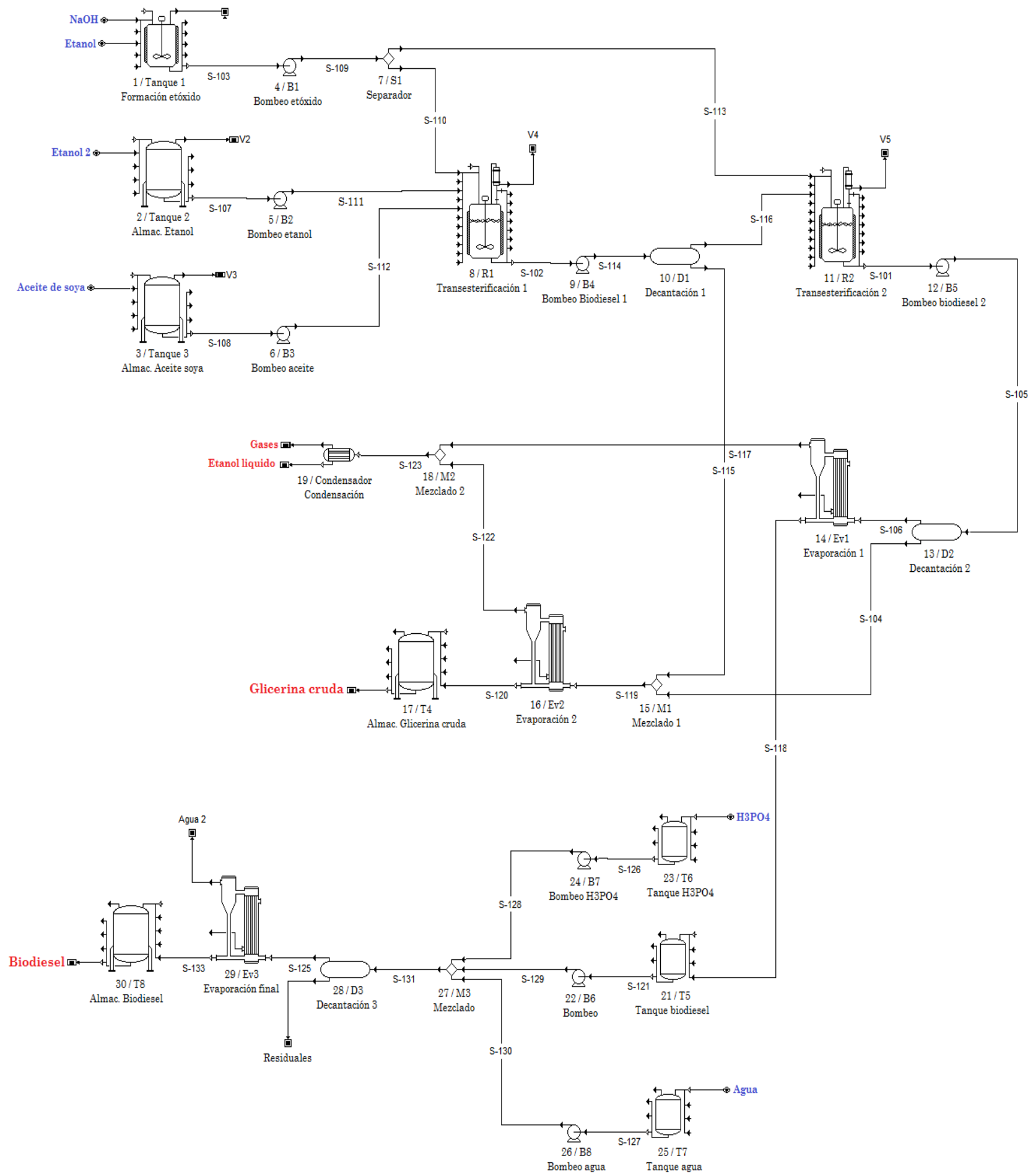

Fig. 4. Simulación del Caso Base en el SuperPro Designer ${ }^{\circledR}$

Fuente: elaboración propia. 
Estudio técnico-económico de dos tecnologías de producción de biodiesel a partir de aceite de soya empleando el simulador superpro designer

El costo de validación y puesta en marcha se consideró como un $15 \%$ de los Costos Fijos Directos (CFD), mientras que los costos asociados con el Aseguramiento y Control de la Calidad se suponen como un $15 \%$ del Costo Total de la Mano de Obra.

Se tomó en cuenta además que no existe rechazo del producto principal (biodiesel) con el motivo de no cumplir con los parámetros de calidad exigidos, que se gastan alrededor de USD $\$ 20000$ anuales para efectuar operaciones de validación del proceso de producción, y que el costo relacionado con el tratamiento de residuales es de un $25 \%$ del costo total de operación [39].

La planta utiliza todos los servicios auxiliares comúnmente consumidos en un proceso de este tipo, es decir, agua de enfriamiento, electricidad, vapor de agua, agua caliente (o condensada) y aire comprimido, los costos de dichos servicios se muestran en la Tabla 2. Se consideró además que la misma trabaja once meses al año, con un mes para acometer operaciones de mantenimiento y reparaciones de equipos y sistemas auxiliares. Se aplicó un salario nominal promedio de USD $\$ 6,00 /$ hora para los operarios que trabajan en la planta, y de USD $\$ 10,00 /$ hora para los supervisores y personal de dirección. En la Tabla 3 se muestran los precios de las principales materias primas consumidas y los productos obtenidos durante el proceso productivo [15], [41] [42] los cuales fueron empleados en la simulación del Caso Base.

En la Tabla 4 se muestran los costos de adquisición de cada equipo empleado en el Caso Base, los cuales fueron tomados de varias fuentes y referencias bibliográficas [15] [43]-[47] y actualizados al mes de febrero del 2019 mediante el índice de costo de la revista Chemical Engineering [48]. Por último, se escoge una capacidad de producción de la planta de 80000 toneladas de biodiesel por año [32].

Tabla 2. Servicios auxiliares consumidos por la planta de producción, y su costo unitario. Fuente: elaboración propia.

\begin{tabular}{ll}
\hline \multicolumn{2}{c}{ y su costo unitario. Fuente: elaboracion propia. } \\
\hline Servicio auxiliar & $\begin{array}{l}\text { Costo unitario } \\
\text { (USD \$/t) }\end{array}$ \\
\hline Agua fría & 0,40 \\
Agua de enfriamiento & 0,05 \\
Vapor de agua & 3,50 \\
\hline
\end{tabular}

Tabla 3. Precios de las principales materias primas y productos obtenidos utilizados en la simulación del Caso Base. Fuente: [15] [41] [42].

\begin{tabular}{cc}
\hline Compuesto & Precio (USD \$/kg) \\
\hline Aceite de soya & 0,715 \\
Etanol & 0,746 \\
Hidróxido de sodio & 0,510 \\
Ácido fosfórico & 0,840 \\
Agua & 0,00005 \\
Biodiesel & 1,340 \\
Glicerol crudo & 0,330 \\
\hline
\end{tabular}


Estudio técnico-económico de dos tecnologías de producción de biodiesel a partir de aceite de soya empleando el simulador superpro designer

Tabla 4. Costo del equipamiento principal empleado en el Caso Base Fuente: [15] [43]-[47].

\begin{tabular}{|c|c|c|c|}
\hline Equipo & Características & Cantidad & $\begin{array}{l}\text { Costo total } \\
{[\text { USD } \$]}\end{array}$ \\
\hline Tanque de formación etóxido & $3 \mathrm{~m}^{3}$ & 1 & 11000 \\
\hline Tanque almacenamiento etanol & $18 \mathrm{~m}^{3}$ & 1 & 13000 \\
\hline Tanque aceite de soya & $25 \mathrm{~m}^{3}$ & 2 & 32000 \\
\hline Reactor 1 & $40 \mathrm{~m}^{3}$ & 2 & 262000 \\
\hline Reactor 2 & $40 \mathrm{~m}^{3}$ & 2 & 262000 \\
\hline Bomba centrífuga 1 & $50 \mathrm{~kW}$ & 1 & 14000 \\
\hline Bomba centrífuga 2 & $50 \mathrm{~kW}$ & 1 & 8000 \\
\hline Bomba centrífuga 3 & $50 \mathrm{~kW}$ & 2 & 16000 \\
\hline Decantador 1 & $60 \mathrm{~m}^{3}$ & 1 & 15000 \\
\hline Bomba centrífuga 4 & $50 \mathrm{~kW}$ & 2 & 16000 \\
\hline Bomba centrífuga 5 & $50 \mathrm{~kW}$ & 2 & 16000 \\
\hline Bomba centrífuga 6 & $50 \mathrm{~kW}$ & 2 & 16000 \\
\hline Decantador 2 & $60 \mathrm{~m}^{3}$ & 1 & 15000 \\
\hline Evaporador multiefecto 1 & $50 \mathrm{~m}^{2}$ & 1 & 57000 \\
\hline Evaporador multiefecto 2 & $50 \mathrm{~m}^{2}$ & 1 & 57000 \\
\hline Condensador & $10 \mathrm{~m}^{2}$ & 1 & 19000 \\
\hline Tanque glicerol crudo & $8 \mathrm{~m}^{3}$ & 1 & 8000 \\
\hline Tanque de ácido fosfórico & $2 \mathrm{~m}^{3}$ & 1 & 11000 \\
\hline Tanque de recepción biodiesel & $25 \mathrm{~m}^{3}$ & 2 & 32000 \\
\hline Tanque de agua & $70 \mathrm{~m}^{3}$ & 2 & 64000 \\
\hline Tanque de biodiesel & $20 \mathrm{~m}^{3}$ & 2 & 28000 \\
\hline Bomba centrífuga 7 & $50 \mathrm{~kW}$ & 1 & 14000 \\
\hline Bomba centrífuga 8 & $48 \mathrm{~kW}$ & 2 & 16000 \\
\hline Evaporador multiefecto 3 & $50 \mathrm{~m}^{2}$ & 1 & 57000 \\
\hline Decantador 3 & $60 \mathrm{~m}^{3}$ & 1 & 30000 \\
\hline Otros equipos & - & - & 466000 \\
\hline Total & & & 1555000 \\
\hline
\end{tabular}

\subsection{Simulación de la Variante en el simulador SuperPro Designer ${ }^{\circledR}$}

Para efectuar la simulación de la Variante en el simulador SuperPro Designer $^{\circledR}$ se tomaron en cuenta todas las condiciones, parámetros y variables técnico-económicas establecidos en el Caso Base, adicionándole solamente los equipos utilizados durante las operaciones de purificación del glicerol (Fig. 5), y tomando en cuenta un precio de venta del glicerol purificado $(99,75 \%$ de pureza) de USD $\$ 0,79 / \mathrm{kg}$ [41]. También se ajustó el tiempo de procesamiento de un lote en la Variante para que durara el mismo tiempo que demora en procesarse un lote de biodiesel en el Caso Base, ya que se consideró que las operaciones de purificación del glicerol ocurren de forma continua y simultánea a las operaciones de refinamiento del biodiesel, con tiempos de duración muy semejantes entre sí. Por último, en la Tabla 5 se muestra el costo de adquisición del equipamiento principal empleado en la Variante [15], [43]-[47], el cual fue actualizado al mes de febrero del 2019 por medio del índice de costo de la revista Chemical Engineering [48]. 
Estudio técnico-económico de dos tecnologías de producción de biodiesel a partir de aceite de soya empleando el simulador superpro designer

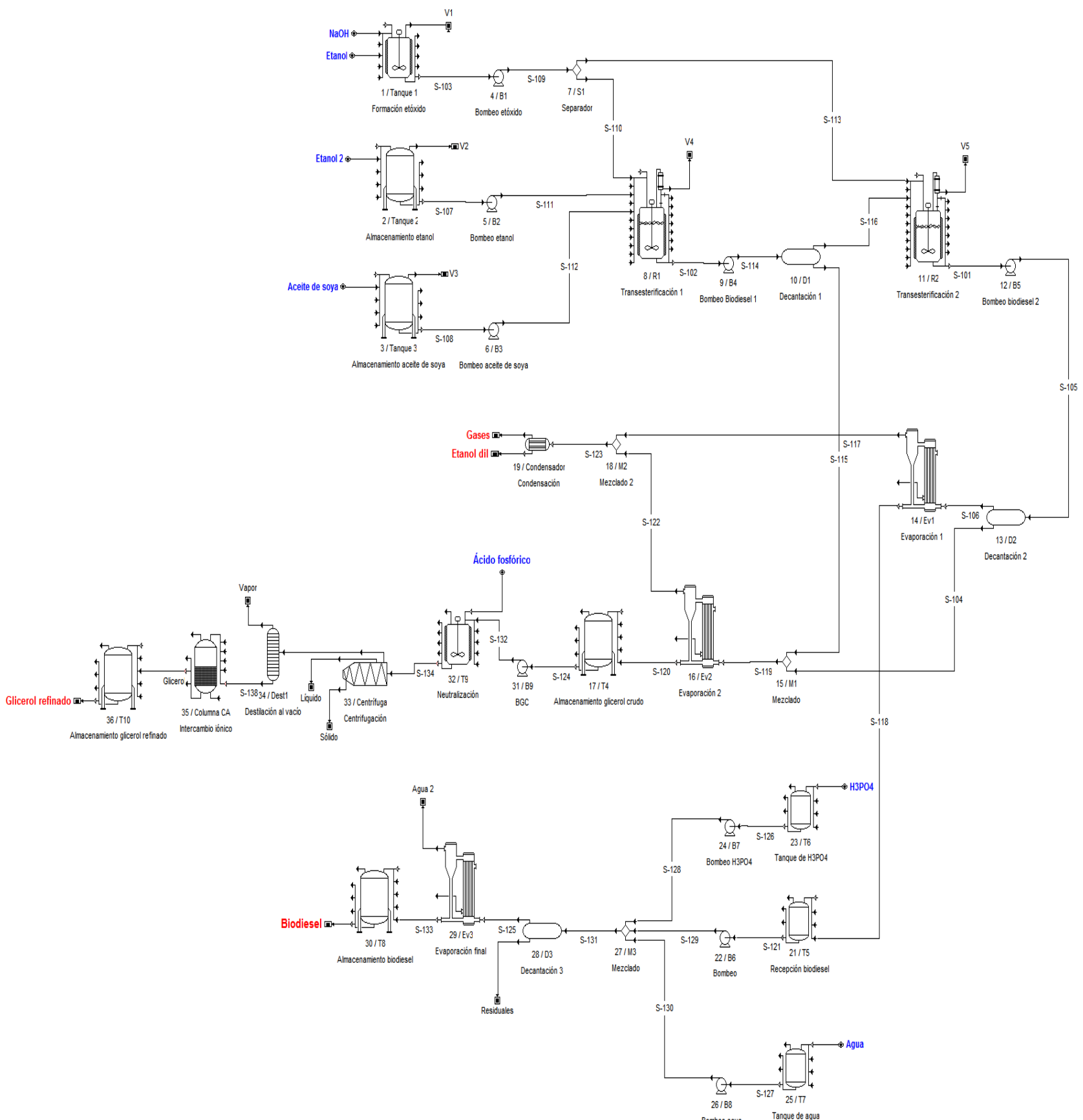

Fig. 5. Simulación de la Variante en el SuperPro Designer ${ }^{\circledR}$

Fuente: elaboración propia. 
Estudio técnico-económico de dos tecnologías de producción de biodiesel a partir de aceite de soya empleando el simulador superpro designer

Tabla 5. Costo del equipamiento principal empleado en la Variante. Fuente: [15] [43]-[47].

\begin{tabular}{|c|c|c|c|}
\hline Equipo & Características & Cantidad & $\begin{array}{l}\text { Costo total } \\
\text { [USD } \$]\end{array}$ \\
\hline Tanque de formación etóxido & $3 \mathrm{~m}^{3}$ & 1 & 11000 \\
\hline Tanque almacenamiento etanol & $18 \mathrm{~m}^{3}$ & 1 & 13000 \\
\hline Tanque aceite de soya & $40 \mathrm{~m}^{3}$ & 2 & 32000 \\
\hline Reactor 1 y 2 & $40 \mathrm{~m}^{3}$ & 4 & 524000 \\
\hline Bomba centrífuga 1 & $50 \mathrm{~kW}$ & 1 & 14000 \\
\hline Bomba centrífuga 2 & $50 \mathrm{~kW}$ & 1 & 8000 \\
\hline Bomba centrífuga 3 & $50 \mathrm{~kW}$ & 2 & 16000 \\
\hline Decantador 1 & $60 \mathrm{~m}^{3}$ & 1 & 15000 \\
\hline Bomba centrífuga 4 & $50 \mathrm{~kW}$ & 2 & 16000 \\
\hline Bomba centrífuga 5 & $50 \mathrm{~kW}$ & 2 & 16000 \\
\hline Bomba centrífuga 6 & $50 \mathrm{~kW}$ & 2 & 16000 \\
\hline Decantador 2 & $60 \mathrm{~m}^{3}$ & 1 & 15000 \\
\hline Evaporador multiefecto 1 & $50 \mathrm{~m}^{2}$ & 1 & 57000 \\
\hline Evaporador multiefecto 2 & $50 \mathrm{~m}^{2}$ & 1 & 57000 \\
\hline Condensador & $10 \mathrm{~m}^{2}$ & 1 & 19000 \\
\hline Tanque glicerol crudo & $8 \mathrm{~m}^{3}$ & 1 & 8000 \\
\hline Tanque de ácido fosfórico & $2 \mathrm{~m}^{3}$ & 1 & 11000 \\
\hline Tanque de recepción biodiesel & $25 \mathrm{~m}^{3}$ & 2 & 32000 \\
\hline Tanque de agua & $70 \mathrm{~m}^{3}$ & 2 & 64000 \\
\hline Tanque de biodiesel & $20 \mathrm{~m}^{3}$ & 2 & 28000 \\
\hline Bomba centrífuga 7 & $50 \mathrm{~kW}$ & 1 & 14000 \\
\hline Bomba centrífuga 8 & $48 \mathrm{~kW}$ & 2 & 16000 \\
\hline Evaporador multiefecto 3 & $50 \mathrm{~m}^{2}$ & 1 & 57000 \\
\hline Decantador 3 & $60 \mathrm{~m}^{3}$ & 1 & 30000 \\
\hline Bomba centrífuga 9 & $50 \mathrm{~kW}$ & 1 & 8000 \\
\hline Tanque de neutralización & $10 \mathrm{~m}^{3}$ & 1 & 10000 \\
\hline Centrífuga decantadora & $150 \mathrm{~m}^{3} / \mathrm{h}$ & & 46000 \\
\hline Columna de destilación al vacío & Diámetro: 3 m & 1 & 270000 \\
\hline Columna carbón activado & Diámetro: 0,7 m & 3 & 9000 \\
\hline Tanque glicerol refinado & $8 \mathrm{~m}^{3}$ & & 8000 \\
\hline Otros equipos & - & - & 617000 \\
\hline Total & & & 2057000 \\
\hline
\end{tabular}

\subsection{Evaluación de la sensibilidad de los indicadores seleccionados para la Variante}

Considerando los resultados técnicoeconómicos obtenidos durante la simulación de la Variante a través del simulador SuperPro Designer ${ }^{\circledR}$, se desarrolló un diseño de experimentos estadístico del tipo Superficie de Respuesta por medio del software estadístico Statgraphics Centurion ${ }^{\circledR}$ v. XVI, con el fin de evaluar la influencia de cinco variables iniciales o de entrada: 1) capacidad de producción de biodiesel; 2) precio de compra del aceite; 3) precio de compra del etanol; 4) precio de venta del biodiesel y 5) precio de venta de la glicerina refinada) sobre tres importantes parámetros de salida de proceso: VAN, TIR y PRI. De esta 

simulador superpro designer

manera se llevará a cabo un estudio de sensibilidad conteniendo cinco entradas y tres salidas.

Para elaborar el diseño de experimentos descrito, a los valores utilizados durante la simulación de la Variante para las cinco variables iniciales consideradas se le aumentará o disminuirá un $20 \%$, para de esta manera tomar en cuenta posibles variaciones $\mathrm{u}$ oscilaciones de los valores que puedan presentar estas variables en el futuro, con el objetivo de evaluar la potencial influencia que puedan presentar estas variaciones en los resultados a obtener del VAN, TIR y PRI, así como también con el fin de seleccionar la corrida (o escenario) más factible desde el punto de vista económico.

Se elaboró un diseño de experimentos del tipo Superficie de Respuesta aleatorizado aplicando la opción "DraperLin small composite design" contenida en el paquete estadístico Statgraphics ${ }^{\circledR}$, a partir del cual se obtuvieron inicialmente 24 corridas en total, las cuales fueron posteriormente optimizadas aplicando la herramienta "D-Optimality" contenida en el propio software estadístico, con el objetivo de seleccionar aquellas corridas que presentan mayor influencia estadística sobre las tres variables de salida tomadas en cuenta, así como también reducir la extensión del estudio de sensibilidad, llegando finalmente a doce corridas.
La Tabla 6 muestra los valores que deben presentar cada una de las cinco variables de entrada tomando en cuenta el rango de $\pm 20 \%$, mientras que la Tabla 7 expone los valores que deberán presentar estos cinco parámetros iniciales dentro del diseño de experimentos optimizado conteniendo las doce corridas.

\subsection{Evaluación de la correlación estadística entre las variables de entrada y los indicadores VAN, TIR y PRI}

Una vez efectuado el estudio de sensibilidad, se procedió a determinar la correlación estadística existente entre las cinco variables de entrada consideradas y los tres parámetros económicos de salida evaluados, con el objetivo de obtener correlaciones o ecuaciones que describan, de forma cuantitativa, la relación estadística existente entre las variables de entrada y salida. Lo anterior se llevó a cabo utilizando la opción "Multiple Regression" contenida en el software estadístico Statgraphics Centurion XVI.

Por último, se determinó la corrida (o escenario) con el resultado económico más positivo con respecto a los resultados de VAN, TIR y PRI obtenidos, además de aquella con el más negativo.

Tabla 6. Valores que deben presentar cada una de las cinco variables de entrada considerando un rango de variación de $\pm 20 \%$. Fuente: elaboración propia.

\begin{tabular}{lccc}
\hline \multicolumn{1}{c}{ Parámetro } & $\begin{array}{c}\text { Valor utilizado en la } \\
\text { Variante }\end{array}$ & $\begin{array}{c}\text { Valor mínimo } \\
(-20 \%)\end{array}$ & $\begin{array}{c}\text { Valor máximo } \\
(+20 \%)\end{array}$ \\
\hline $\begin{array}{l}\text { Capacidad de producción (t/año) } \\
\text { Precio de compra aceite de soya (USD }\end{array}$ & 80000 & 64000 & 96000 \\
$\$ / \mathrm{kg})$ & 0,879 & 0,70 & 1,05 \\
Precio de compra etanol (USD \$/kg) & 0,7461 & 0,60 & 0,90 \\
Precio de venta biodiesel (USD \$/kg) & 1,33 & 1,06 & 1,60 \\
Precio de venta glicerina refinada (USD & 0,79 & 0,63 & 0,95 \\
$\$ / \mathrm{kg})$ & & & \\
\hline
\end{tabular}


Estudio técnico-económico de dos tecnologías de producción de biodiesel a partir de aceite de soya empleando el simulador superpro designer

Tabla 7. Valores que deberán presentar los cinco parámetros iniciales en el diseño de experimentos optimizado. Fuente: elaboración propia.

\begin{tabular}{cccccc}
\hline Corrida & $\begin{array}{c}\text { Capacidad } \\
(\mathrm{t} / \mathrm{a})\end{array}$ & $\begin{array}{c}\text { Precio aceite } \\
(\text { USD } \$ / \mathrm{kg})\end{array}$ & $\begin{array}{r}\text { Precio etanol } \\
\text { (USD } \$ / \mathrm{kg})\end{array}$ & $\begin{array}{c}\text { Precio biodiesel } \\
\text { (USD } \$ / \mathrm{kg})\end{array}$ & $\begin{array}{c}\text { Precio glicerina } \\
\text { refinada (USD } \\
\$ / \mathrm{kg})\end{array}$ \\
\hline 1 & 96000 & 1,05 & 0,60 & 1,06 & 0,63 \\
2 & 96000 & 0,70 & 0,90 & 1,60 & 0,63 \\
3 & 96000 & 1,05 & 0,90 & 1,06 & 0,63 \\
4 & 96000 & 0,70 & 0,90 & 1,60 & 0,95 \\
5 & 64000 & 0,70 & 0,60 & 1,06 & 0,63 \\
6 & 64000 & 0,70 & 0,90 & 1,06 & 0,95 \\
7 & 96000 & 0,70 & 0,60 & 1,06 & 0,95 \\
8 & 64000 & 0,70 & 0,60 & 1,60 & 0,63 \\
9 & 64000 & 1,05 & 0,90 & 1,06 & 0,95 \\
10 & 64000 & 1,05 & 0,90 & 1,60 & 0,63 \\
11 & 64000 & 1,05 & 0,60 & 1,60 & 0,95 \\
12 & 96000 & 1,05 & 0,90 & 1,60 & 0,95 \\
\hline
\end{tabular}

\section{RESULTADOS Y DISCUSIÓN}

\subsection{Comparación de los resultados obtenidos para ambas propuestas tecnológicas}

La Tabla 8 presenta una comparación de los principales indicadores técnicoeconómicos obtenidos para las dos propuestas tecnológicas evaluadas en el simulador SuperPro Designer ${ }^{\circledR}$, mientras que la Tabla 9 compara los resultados obtenidos de las principales partidas incluidas en los costos de operación, así como sus porcientos de ocupación, también para las dos propuestas tecnológicas consideradas.

Según los resultados mostrados en la tabla anterior, para una planta con una inversión inicial de USD \$18455000, el Caso Base se puede calificar de económicamente rentable y confiable desde el punto de vista inversionista, ya que el PRI no es superior a cinco años (2,54 años), la TIR es de $33,83 \%$ y el VAN tiene resultado positivo (USD $\$ 17444000$ ), lo cual es un indicativo de rentabilidad de la inversión y ganancias positivas [46] [47] [49].
Los resultados mostrados en la Tabla 8 permiten concluir que implementar operaciones de purificación de glicerol dentro del proceso de producción del biodiesel también resulta factible desde el punto de vista económico, ya que los principales indicadores de factibilidad $y$ rentabilidad considerados presentan valores evidentemente positivos.

De esta manera, tomando en cuenta que el PRI es inferior a cinco años $(2,26$ años), la TIR es de $38,05 \%$, y el VAN presenta valor positivo (USD $\$ 22577000$ ), se puede calificar al proceso de fabricación de biodiesel conteniendo operaciones de purificación de glicerol como rentable y viable desde el punto de vista económico e inversionista [46] [47] [49]. Según se puede apreciar en la Tabla 7, tanto la inversión total de capital como los costos de operación se incrementaron en un 13,70\% y $2,85 \%$ respectivamente para la Variante en comparación con el Caso Base. Lo anterior se debe a que en la Variante se tiene que adquirir una cantidad adicional de equipamiento para efectuar las operaciones de purificación del glicerol crudo obtenido, con la consiguiente inversión añadida en tuberías, 
Estudio técnico-económico de dos tecnologías de producción de biodiesel a partir de aceite de soya empleando el simulador superpro designer

instrumentación, instalaciones eléctricas y servicios auxiliares, entre otros, además de consumir cantidades adicionales de agua y ácido fosfórico como materias primas, así como también de necesitar mayor cantidad de mano de obra (operarios, supervisores, etc.) para efectuar las operaciones propias de los equipos adquiridos, con el consiguiente incremento de los costos por salario y existir mayor consumo de energía eléctrica y servicios auxiliares.

Igualmente se incrementa el costo relacionado con la arrancada y puesta en marcha en un $23,08 \%$, lo cual tiene su explicación en la mayor cantidad de equipos que deben ser validados desde el punto de vista operacional antes de proceder a la fabricación comercial, mientras que el capital de trabajo también

Tabla 8. Comparación de los principales indicadores técnico-económicos obtenidos tanto para el Caso Base como la Variante. Fuente: elaboración propia.

\begin{tabular}{lcc}
\hline Parámetro & Caso Base & Variante \\
\hline Inversión total de capital [USD \$] & 18455000 & 21384000 \\
Costo de operación [USD \$/año] & 106118000 & 109236000 \\
Ganancias totales anuales [USD \$/año] & 113349000 & 118125000 \\
Capital de trabajo [USD \$] & 9540000 & 9793000 \\
Arrancada y puesta en marcha [USD \$] & 1163000 & 1512000 \\
Cantidad de biodiesel producido [t/a] & 83232 & 83232 \\
Cantidad de glicerol crudo producido [t/a] & 18407 & - \\
Cantidad de glicerol purificado producido [t/a] & - & 13222 \\
Tiempo que demora un lote [h/lote] & 19 & 19 \\
Cantidad de lotes por año [lotes/a] & 2380 & 2380 \\
Ganancias anuales por venta de biodiesel [USD \$/año] & 107704000 & 107704000 \\
Ganancias anuales por venta de glicerol [USD \$/año] & 5645000 & 10421000 \\
Costo unitario de producción [USD \$/kg] & 1,27 & 1,31 \\
Margen bruto [\%] & 6,38 & 7,53 \\
Retorno de la Inversión [\%] & 39,44 & 44,19 \\
Período de Retorno de la Inversión (PRI) [años] & 2,54 & 2,26 \\
Tasa Interna de Retorno (TIR) [\%] & 33,83 & 38,05 \\
Valor Actual Neto ( van) [USD \$] & 17444000 & 22577000 \\
\hline
\end{tabular}

Tabla 9. Resultados económicos de las principales partidas que influyen en los costos de operación tanto para el Caso Base como la Variante. Fuente: elaboración propia.

\begin{tabular}{lcccc}
\hline Partida & $\begin{array}{c}\text { Caso Base } \\
\text { [USD } \$ / \text { año] }\end{array}$ & $\%$ & $\begin{array}{c}\text { Variante } \\
\text { [USD \$/año] }\end{array}$ & $\%$ \\
\hline Materias primas & 102662000 & 96,74 & 104709000 & 95,86 \\
Mano de obra & 1922000 & 1,81 & 2400000 & 2,20 \\
Depreciación de la instalación & 874000 & 0,82 & 1137000 & 1,04 \\
Control/aseguramiento de la calidad & 288000 & 0,27 & 360000 & 0,33 \\
Servicios auxiliares & 331000 & 0,31 & 590000 & 0,54 \\
Gastos misceláneos & 20000 & 0,02 & 20000 & 0,02 \\
Promoción y marketing & 20000 & 0,02 & 20000 & 0,02 \\
Total & 106117000 & 100 & 109236000 & 100 \\
\hline
\end{tabular}



simulador superpro designer

aumenta en un 2,58\% para la Variante con respecto al Caso Base, lo cual se debe a un mayor gasto por concepto de mano de obra (salario), electricidad, materias primas (principalmente agua y ácido fosfórico), servicios auxiliares (vapor de agua, agua de enfriamiento), tratamiento de residuales, control y aseguramiento de la calidad, entre otros elementos involucrados en esta partida de costo.

Por otro lado, el Margen Bruto se incrementó en un 1,15\%, el Retorno de la Inversión aumentó en $4,75 \%$, la Tasa Interna de Retorno creció un 4,22\%, el Valor Actual Neto se incrementó en un $22,74 \%$ (alrededor de USD \$5,13 millones) y el Período de Recuperación de la Inversión se redujo en 0,28 años para la Variante con respecto al Caso Base.

Lo anterior se debe fundamentalmente a un incremento de las ganancias totales anuales en un 4,04\% (alrededor de USD $\$ 4,8$ millones anuales) producto al aumento del precio de venta del glicerol desde USD $\$ 0,33 / \mathrm{kg}$ para el glicerol crudo (70-80\% de pureza) hasta USD $\$ 0,79 / \mathrm{kg}$ para el glicerol refinado (99,7\% de pureza), manteniéndose constante la cantidad de biodiesel a obtener para ambas tecnologías en alrededor de 80000 toneladas anuales.

Lo anterior se puede apreciar desde otro punto de vista comparando las ganancias anuales obtenidas por concepto de venta de glicerol, las cuales tienen un valor de USD \$5645000 para el Caso Base, y de USD \$10421000 para la Variante, es decir, se incrementa alrededor de 1,84 veces en esta última, lo cual influye positivamente en la rentabilidad económica del proceso global. De esta manera se puede apuntar que el incremento de los costos capitales y de operación acontecidos en la Variante debido a la instalación de operaciones y procesos adicionales de purificación del glicerol crudo no influye negativamente en los principales parámetros técnicoeconómicos del proceso global, ya que el aumento de los ingresos totales anuales obtenidos por concepto de venta del glicerol refinado resulta suficiente para calificar el proyecto tecnológico como económicamente rentable y factible desde el punto de vista inversionista, validando de esta manera la factibilidad técnico-económica de la Variante propuesta.

En la Tabla 9 se puede observar que la partida que más influye en los costos anuales de operación para las dos tecnologías evaluadas son las Materias Primas, con más del $95 \%$ para ambas, mientras que los gastos relativos a la mano de obra y la depreciación de la instalación constituyen las otras dos partidas de mayor influencia en este parámetro económico.

También se puede distinguir en la Tabla 9 que todas las partidas consideradas en los costos anuales de operación presentan valores superiores para la Variante con respecto al Caso Base.

Esto tiene su justificación en la mayor cantidad de equipos utilizados en la primera mencionada, lo cual trae consigo un mayor gasto por mano de obra (salario) para operar los mismos, mayor consumo de servicios auxiliares (vapor de agua, agua de enfriamiento, electricidad) y materias primas (fundamentalmente agua y ácido fosfórico), así como también un incremento de los gastos relacionados con el control y aseguramiento de la calidad y aquellos correspondientes a la depreciación de la planta.

\subsection{Evaluación del estudio de sensibilidad efectuado con respecto a las variables iniciales seleccionadas}

La Fig. 6 presenta los resultados del estudio de sensibilidad efectuado para las 12 corridas consideradas. Un análisis del mismo permite establecer que la corrida \#4 presenta el escenario económico más optimista y positivo en cuanto a los mayores valores de VAN y TIR, y menor valor de PRI obtenidos, con USD $\$ 184267000, \quad 307,11 \%$ y 0,42 años, respectivamente. 
Estudio técnico-económico de dos tecnologías de producción de biodiesel a partir de aceite de soya empleando el simulador superpro designer

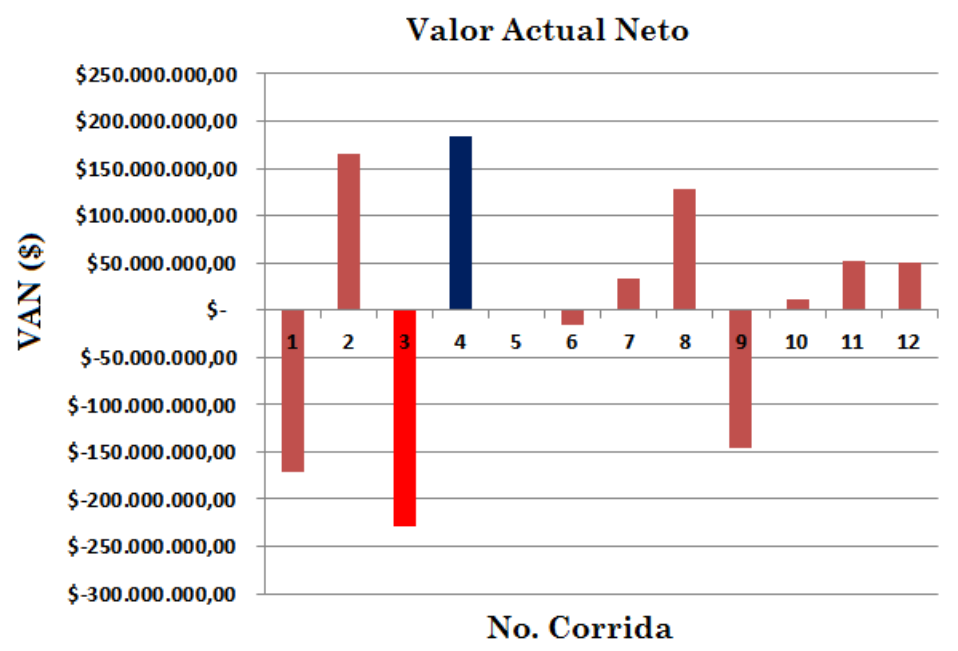

a)

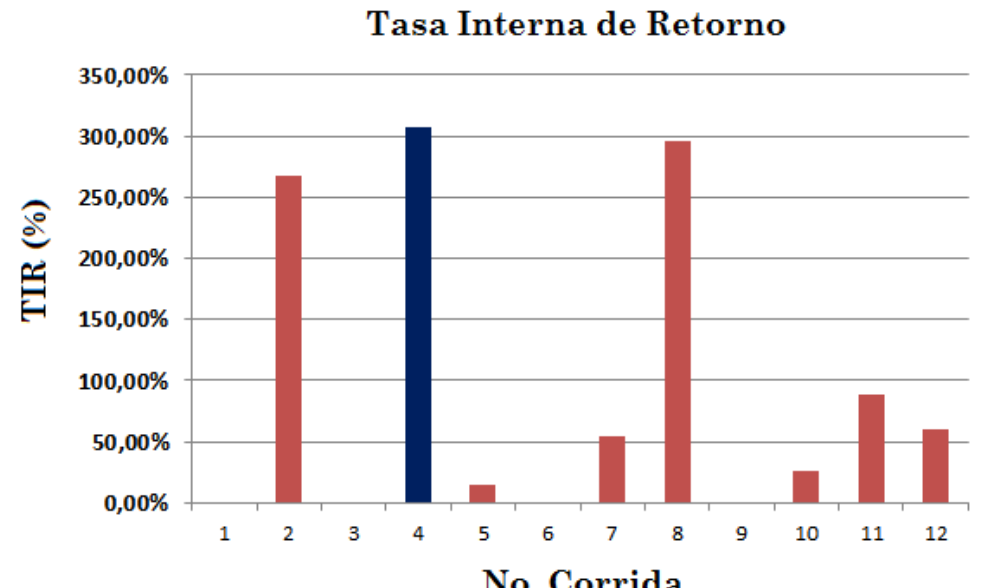

No. Corrida

b)

Período de Recuperación de la Inversión

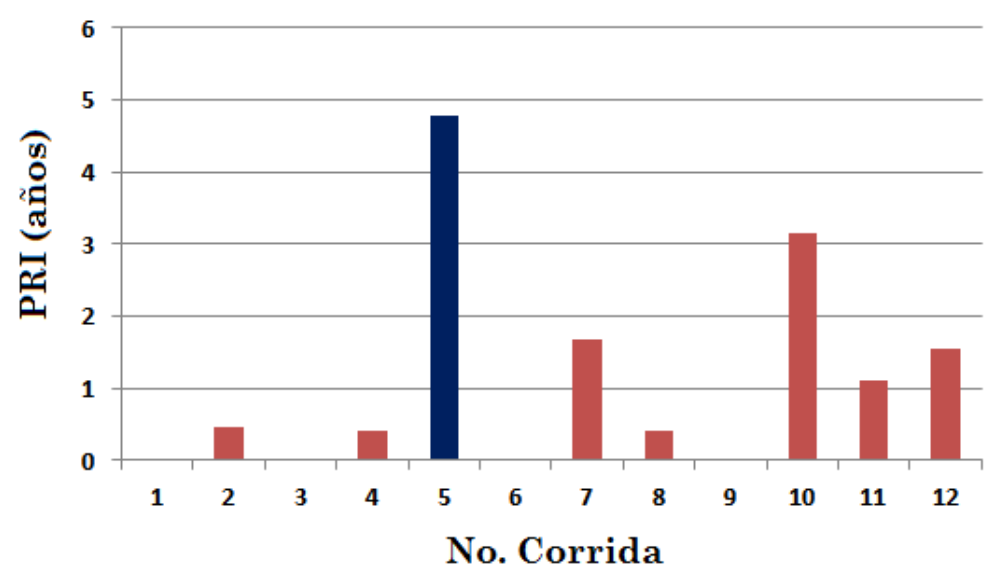

c)

Fig. 6. Resultados obtenidos para cada una de las corridas incluidas en el estudio de sensibilidad efectuado. a) Valor actual neto, b) Tasa Interna de Retorno, c) Periodo de Recuperación de la Inversión

Fuente: elaboración propia 
Esto es debido a que esta corrida presenta la mayor capacidad de producción anual de biodiesel (96000 t/a), con el mayor precio de venta del mismo (USD $\$ 1,60 / \mathrm{kg}$ ) y la glicerina refinada (USD $\$ 0,95 / \mathrm{kg}$ ); así como también con el menor precio de compra del aceite de soya (USD $\$ 0,70 / \mathrm{kg}$ ), el cual constituye la materia prima que más influencia presenta en los costos de operación.

Es decir, se produce a la mayor capacidad de producción posible adquiriendo la principal materia prima consumida en el proceso (aceite de soya) al menor costo posible, y vendiendo los dos productos obtenidos (biodiesel y glicerina refinada) al mayor precio de venta posible, lo cual influye positivamente en la rentabilidad global del proceso productivo.

Por otro lado, la corrida \#3 fue la que tuvo el peor escenario económico de todas, con un valor del VAN de USD \$229499000, sin presentar valores viables de TIR y PRI.

Lo anterior se debe a que en esta corrida se obtiene el mayor costo de las dos materias primas consideradas (aceite de soya con USD $\$ 1,05 / \mathrm{kg}$ y etanol con USD $\$ 0,90 / \mathrm{kg})$, así como el menor precio de venta del biodiesel (USD $\$ 1,06 / \mathrm{kg}$ ) y de glicerina refinada (USD $\$ 0,63 / \mathrm{kg}$ ) para una capacidad de producción de la planta de 96000 t/a.

\subsection{Resultados obtenidos con respecto a la correlación estadística existente entre las 5 variables de entrada y los indicadores van, tir y pri}

$\mathrm{Al}$ efectuar la evaluación de la correlación estadística existente entre las cinco variables de entrada consideradas (capacidad de producción de biodiesel de la planta; precio de compra del aceite de soya; precio de compra del etanol; precio de venta del biodiesel y precio de venta de glicerol refinado) sobre tres indicadores económicos importantes del proceso (VAN, TIR y PRI), se obtuvo una ecuación multi-factorial para cada uno de los tres indicadores evaluados, las cuales se muestran a continuación:

-Valor Actual Neto (VAN):

VAN $=-1,50405 \mathrm{E} 8+129,515 x$ Capacidad - 4,33515E8xPrecio de compra aceite de soya - 6,98228E7xPrecio de compra etanol $+3,51037 \mathrm{E} 8 \mathrm{xPrecio}$ de venta biodiesel $+1,41521 \mathrm{E} 8 \mathrm{xPrecio}$ de venta glicerina refinada.

-Tasa Interna de Retorno (TIR):

TIR $=-65,4901$

0,0000381458xCapacidad

701,697xPrecio de compra aceite de soya - 84,5733xPrecio de compra etanol + $517,938 x$ Precio de venta biodiesel + $120,91 x$ Precio de venta glicerina refinada.

-Período de Recuperación de la Inversión (PRI):

$\mathrm{PRI}=10,7084-0,00007175 \mathrm{xCapacidad}+$ $2,15429 x$ Precio de compra aceite de soya $+7,87333 x$ Precio de compra etanol 7,40741xPrecio de venta biodiesel $0,15 x$ Precio de venta glicerina refinada.

Estos modelos permiten estimar los principales indicadores dinámicos de la inversión para diferentes variaciones de precios de las materias primas, considerando la incertidumbre. De esta forma, los productores de biodiesel con aceite de soya pueden realizar predicciones del comportamiento económico de la planta para diferentes escenarios de las cinco variables iniciales consideradas.

\section{CONCLUSIONES}

La simulación de ambas propuestas tecnológicas de producción de biodiesel en el simulador SuperPro Designer ${ }^{\circledR}$ permitió conocer sus principales indicadores técnicoeconómicos. Esta herramienta permitió realizar un estudio de sensibilidad considerando diferentes variables técnico- 
Estudio técnico-económico de dos tecnologías de producción de biodiesel a partir de aceite de soya empleando el simulador superpro designer

económicas del proceso, facilitando examinar varias alternativas y conocer aquella con los mejores indicadores de rentabilidad.

Se necesitan USD $\$ 18455000$ y USD $\$ 21384000$ para construir el Caso Base y la Variante, respectivamente.

Los costos anuales de operación del Caso Base y la Variante alcanzaron valores de USD \$106118000 y USD \$109236000, respectivamente.

El Caso Base presentó valores de los indicadores VAN, TIR y PRI de USD $\$ 17444000, \quad 33,83 \%$ y 2,54 años, respectivamente, mientras que los valores de estos tres indicadores para la Variante fueron de USD $\$ 22577000,38,05 \%$ y 2,26 años, por lo que se puede concluir que ambas propuestas tecnológicas de producción de biodiesel son económicamente rentables $\mathrm{y}$ atractivas desde el punto de vista inversionista, bajo las condiciones económicas actuales de Brasil.

La Variante constituye la propuesta tecnológica más rentable de ambas consideradas.

La implementación de operaciones de purificación de glicerol incrementa la rentabilidad económica global de la planta de producción de biodiesel en un $22,74 \%$ con respecto al valor del VAN y en un $4,22 \%$ con relación al valor de la TIR, mientras que el PRI se reduce en un $11 \%$.

Las ganancias netas anuales para el Caso Base y la Variante alcanzaron valores de USD $\$ 113349000$ y USD \$118 125000 , respectivamente.

La corrida que mejor escenario económico presentó durante el estudio de sensibilidad efectuado fue la número 4 con una VAN = USD \$184267000, una TIR = $307,11 \%$ y un PRI $=0,42$ años, mientras que la de peor resultado económico fue la número 3 con un VAN = USD \$ 229499000 .

Se obtuvieron correlaciones que relacionan los parámetros VAN, TIR y PRI con cinco variables iniciales o de entrada del proceso productivo.

\section{REFERENCIAS}

[1] L. D. Fernández Betancurt, "Energías alternativas", TecnoLógicas, no. 14, pp. 105126, Jun. 2005.

https://doi.org/10.22430/22565337.538

[2] S. D. Romano y P. A. Sorichetti, Dielectric Spectroscopy in Biodiesel Production and Characterization. London, United Kingdom: Springer-Verlag London Limited, 2011.Disponible en: URL

[3] J. I. Torregrosa, Conceptos básicos de simulación de procesos. Valencia, España: Departamento de Ingeniería Química y Nuclear, Universitat Politècnica de València, 2013. Disponible en: URL

[4] N. A. Auli, M. Sakinah, A. M. M. A. Bakri, H. Kamarudin, and M. N. Norazian, "Simulation Of Xylitol Production: A Review", Australian Journal of Basic and Applied Sciences, vol. 7, no. 5, pp. 366-372, 2013. Disponible en: URL

[5] S. Mani, J. Sundaram y K. C. Das, "Process simulation and modeling: Anaerobic digestion of complex organic matter", Biomass and Bioenergy, vol. 93, pp. 158-167, Oct 2016. https://dx.doi.org/10.1016/j.biombioe.2016.07.0 $\underline{18}$

[6] A. Csighy, A. Koris y G. Vatai, "Modelling the Partial Demineralization Process of Cow Milk by Superpro Designer", Hungarian Journal of Industry and Chemistry, vol. 45, n. $^{\circ} 2$, pp. 9$12,2017$.

https://doi.org/10.1515/hjic-2017-0013

[7] A. C Aguiar, J. F. Osorio-Tobón, L. P. Sales Silva, G. Fernandez Barbero y J. Martínez, "Economic analysis of oleoresin production from malagueta peppers (Capsicum frutescens) by supercritical fluid extraction", The Journal of Supercritical Fluids, vol. 133, pp. 86-93, Mar. 2018. https://doi.org/10.1016/j.supflu.2017.09.031

[8] P. Somavat, D. Kumar y V. Singh, "Technoeconomic feasibility analysis of blue and purple corn processing for anthocyanin extraction and ethanol production using modified dry grind process", Industrial Crops \& Products, vol. 115, pp. 78-87, May. 2018 https://doi.org/10.1016/j.indcrop.2018.02.015

[9] D. K. Govindarajan, Y. Meganathan, G. P. Udayakumar y R. Kothandan, "TechnoEconomic Analysis for the Production of Ethanol from Ipomoea batatas (sweet potato)", American International Journal of Research in Science, Technology, Engineering \& Mathematics, pp. 13-24, 2019. Disponible en: URL

[10] SuperPro Designer ${ }^{\circledR}$, "Biodiesel production from degummed soybean oil”, Intelligen, Inc., 
Estudio técnico-económico de dos tecnologías de producción de biodiesel a partir de aceite de soya empleando el simulador superpro designer

\& Massachusetts Institute of Technology, 2005. Disponible en: URL

[11] C. M. García, "Simulación de una planta de producción y purificación de biodiesel a partir de aceite de palma y etanol usando el simulador comercial HYSYs Plant 3.2", (Tesis de grado), Facultad de Ingenierías Fisicoquímicas, Universidad Industrial de Santander, Bucaramanga, Colombia, 2007. Disponible en: URL

[12] S. Lee, D. Posarac y N. Ellis, "Process simulation and economic analysis of biodiesel production processes using fresh and waste vegetable oil and supercritical methanol", Chemical Engineering Research and Design, vol. 89, no. 12. pp. 2626-2642, Dec. 2011. https://doi.org/10.1016/j.cherd.2011.05.011

[13] V. F. Marulanda, "Biodiesel production by supercritical methanol transesterification: Process simulation and potential environmental impact assessment", Journal of Cleaner Production, vol. 33, pp. 109-116, Sep. 2012.

https://doi.org/10.1016/j.jclepro.2012.04.022

[14] M. Franco, "Simulación del proceso de producción de biodiesel a partir de aceites vegetales en condiciones súper-críticas", (Tesis de Maestría), Universitat Politecnica de Catalunya, Barcelona, 2013. Disponibe en: $\underline{\mathrm{URL}}$

[15] A. F. Young, F. L. P. Pessoa y E. M. Queiroz, "Comparison between Biodiesel Production from Soybean Oil and Palm Oil with Ethanol: Design and Economic Evaluation", Chemical Engineering Transactions, vol. 43, pp. 325-330, May. 2015.

https://doi.org/10.3303/CET1543055

[16] L. R. D. la Rosa, E. Henríquez Montero, E. Sánchez Tuirán y K. A. Ojeda Delgado, "Diseño y simulación de una planta para la producción de biodiésel a partir de Jatropha curcas L. en el departamento de Bolívar", rev.ion, vol. 28, no. 1, pp. 73-85. Jul. 2015. Disponible en: URL

[17] Okullo y N. Tibasiima, "Process Simulation of Biodiesel Production from Jatropha Curcas Seed Oil", American Journal of Chemical Engineering, vol. 5, n. ${ }^{\circ}$ 4, pp. 56-63, Jul. 2017. https://doi.org/10.11648/j.ajche.20170504.12

[18] M. I. M. Hamid y K. M. Wagialla, "Simulation of Jatropha Biodiesel production using SuperPro Designer", Journal of Basic and Applied Science, vol. 2, no. 1, pp. 115-142, Mar. 2017. Disponible en: URL

[19] Y. B. Abdurakhman, Z. A. Putra y M. R. Bilad, "Aspen HYSYS Simulation for Biodiesel Production from Waste Cooking Oil using Membrane Reactor", en IOP Conf. Series: Materials Science and Engineering, vol. 180, pp. $\quad 1-6, \quad$ Bandung. 2017. https://doi.org/10.1088/1757899X/180/1/012273

[20] S. P. Souza, J. E. A. Seabra y L. A. Horta Nogueira, "Feedstocks for biodiesel production: Brazilian and global perspectives", Biofuels, pp. 1-24, vol. p, no. 4, Jan. 2017. https://dx.doi.org/10.1080/17597269.2017.1278 $\underline{931}$

[21] F. P. Nascimento, A. R. G. Oliveira, M. L. L. Paredes, A. L. H. Costa y F. L. P. Pessoa, "Biodiesel Production from Supercritical Ethanolysis of Soybean Oil", Chemical Engineering Transactions, vol. 32, pp. 829-834, $2013 . \quad$ Disponible en: https://doi.org/10.3303/CET1332139

[22] M. Rahimi, B. Aghel, M. Alitabar, A. Sepahvand y H. R. Ghasempour, "Optimization of biodiesel production from soybean oil in a microreactor", Energy Conversion and Management, vol. 79, pp. 599605, Mar. 2014.

https://doi.org/10.1016/j.enconman.2013.12.065

[23] S. Joshi, P. R. Gogate, P. F. Moreira y R. Giudici, "Intensification of biodiesel production from soybean oil and waste cooking oil in the presence of heterogeneous catalyst using high speed homogenizer". UltrasonicsSonochemistry, vol. 39, pp. 645-653, 2017. https://doi.org/10.1016/j.ultsonch.2017.05.029

[24] P. Adewale, M. J. Dumont y M. Ngadi, "Enzyme-catalyzed synthesis and kinetics of ultrasonic-assisted biodiesel production from waste tallow", Ultrasonics - Sonochemistry, vol. 27, pp. 1-9. Nov. 2015. https://doi.org/10.1016/j.ultsonch.2015.04.032

[25] V. F. De Almeida, P. J. García-Moreno, A. Guadix, y E. M. Guadix, "Biodiesel production from mixtures of waste fish oil, palm oil and waste frying oil: Optimization of fuel properties", Fuel Processing Technology, vol. 133, pp. 152-160, May. 2015. http://dx.doi.org/10.1016/j.fuproc.2015.01.041 [26] E. Alptekin, M. Canakci y H. Sanli, "Biodiesel production from vegetable oil and waste animal fats in a pilot plant", Waste Management, vol. 34, no. 11, pp. 2146-2154, Nov.

2014.

https://doi.org/10.1016/j.wasman.2014.07.019

[27] P. J. García-Moreno, M. Khanum, A. Guadix y E. M. Guadix, "Optimization of biodiesel production from waste fish oil", Renewable Energy, vol. 68, pp. 618-624, Aug. 2014. https://doi.org/10.1016/j.renene.2014.03.014

[28] A. E. da Costa, C. A. Klimeck Gouvea, B. B. Lobo, J. K. Andreazza, K. S. Fadhil Al-Rubaie "Optimization for producing biodiesel from ethanol and waste frying oil with a high concentration of ester", Revista Facultad de Ingenierí, Universidad de Antioquia n. ${ }^{\circ}$ 79, 
Estudio técnico-económico de dos tecnologías de producción de biodiesel a partir de aceite de soya empleando el simulador superpro designer

pp. $\quad 185-191, \quad 2016 . \quad 2006$ http://dx.doi.org/10.17533/udea.redin.n79a17

[29] R. D. Micic, M. D. Tomic, F. E. Kiss, E. B. Nikolic-Djoric y M. Đ. Simikic, "Influence of reaction conditions and type of alcohol on biodiesel yields and process economics of supercritical transesterification", Energy Conversion and Management, vol. 86, pp. 717726, Oct. 2014.

https://doi.org/10.1016/j.enconman.2014.06.052

[30] N. A. Negm, G. H. Sayed, O. I. Habib, F. Z. Yehia y E. A. Mohamed, "Heterogeneous catalytic transformation of vegetable oils into biodiesel in one-step reaction using super acidic sulfonated modified mica catalyst", Journal of Molecular Liquids, vol. 237, pp. 3845 , Jul. 2017. https://doi.org/10.1016/j.molliq.2017.04.076

[31] T. Wang. "Leading biodiesel producers worldwide in 2018, by country (in billion liters)", Disponible en: URL

[32] S. Barros. Biofuels Annual. Sao Paulo, Brasil: Global Agricultural Information Network (GAIN), No. BR18017. Oct. 2018. Disponible en: URL

[33] ChemicaLogic Corporation, "Thermodynamic and Transport Properties of Water and Steam for Windows application available at no cost.", ChemicaLogic $^{\circledR}$ Steam Tab Companion, Version 2.0, 2003. Disponible en: URL

[34] M. Ahmad, M. A. Khan, M. Zafar y S. Sultana, Practical Handbook on Biodiesel Production and Properties, 2nd ed., Boca Raton, USA: CRC Press, 2012. Disponible en: URL

[35] G. Knothe, J. Krahl y J. V. Gerpen, The Biodiesel Handbook, 2nd ed., Urbana, USA: AOCS Press, 2010. Disponible en: URL

[36] A. B. Koc, M. Abdullah y M. Fereidouni, Soybeans Processing for Biodiesel Production. En T.-B. Ng (Ed.), Soybean-Applications and Technology. Rijeka, Croatia: InTech, pp. 19-32. 2011. Disponible en: URL

[37] J. McFarlane, Processing of Soybean Oil into Fuels. In D. Krezhova (Ed.), Recent Trends for Enhancing the Diversity and Quality of Soybean Products. Rijeka, Croatia: InTech, 2011. Disponible en: URL

[38] M. Stoytcheva y G. Montero, Biodiesel Feedstocks and Processing Technologies. Rijeka, Croatia: InTech, 2011. Disponible en: URL

[39] M. J. Haas, A. J. McAloon, W. C. Yee, and T. A. Foglia, "A process model to estimate biodiesel production costs," Bioresour. Technol., vol. 97, no. 4, pp. 671-678, Mar.

[40] Oleofinos. "Especificación técnica Aceite de Soya R.B.D”. 2019. Disponible en: URL

[41] Agencia Nacional do Petróleo, Gas Natural e Biocombustíveis . "Anuário Estatístico Brasileiro do Petróleo, Gás Natural e Biocombustíveis 2019". Recuperado el 16 de mayo, 2019. Disponible en: URL

[42] CEPEA. Center for Advanced Studies in Applied Economics, 2018. Disponible en: URL

[43] Matche. "Chemical Equipment Cost", 2014. Recuperado el 20 de junio de 2019, de Disponible en: URL

[44] R. H. Perry y D. Green, Perry's Chemical Engineers' Handbook, 8th ed. New York, USA: McGraw-Hill, 2008. Disponible en: URL

[45] M. S. Peters, K. D. Timmerhaus y R. E. West, Plant Design and Economics for Chemical Engineers, 4th ed. New York, USA: McGrawHill, 2003.

[46] R. K. Sinnott, Chemical Engineering Design, 4th ed., vol. 6, Oxford, United Kingdom: Elsevier Butterworth-Heinemann, 2005. Disponible en: URL

[47] G. Towler y R. Sinnott, Chemical Engineering Design, 2nd ed. Oxford, United Kingdom: Butterworth-Heinemann, 2013. Disponible en: URL

[48] S. Jenkins, "Facts At your Fingertips, Fermentation Considerations and Economics", Chemical Engineering, vol. 126, n. ${ }^{\circ}$ 6, pp. 72, June 2019. Disponible en: URL

[49] G. Baca Urbina, Evaluación de proyectos. 6ta ed., México, D. F., México: McGrawHill/Interamericana Editores, S.A. de C.V., 2010. Disponible en: URL

\section{CONTRIBUCION DE LOS AUTORES}

(D) 1 Propuso las tecnologías y las metodologías de cálculo, efectuó la simulación, redacción del texto.

(D) 2 Búsqueda bibliográfica, efectuó la simulación, redacción del texto, validación de los resultados.

(D) 3 Estudió de sensibilidad, adquisición de datos, efectuó la simulación.

(i) 4 Redacción y escritura del texto, supervisión de los resultados, elaboración de las figuras. 